\title{
Consumption of whole grains is associated with improved diet quality and nutrient intake in children and adolescents: the National Health and Nutrition Examination Survey 1999-2004
}

\author{
Carol E O'Neil ${ }^{1, *}$, Theresa A Nicklas ${ }^{2}$, Michael Zanovec ${ }^{1}$, Susan $\mathrm{S} \mathrm{Cho}^{3}$ and \\ Ronald Kleinman ${ }^{4}$ \\ 'Louisiana State University Agricultural Center, 261 Knapp Hall, Baton Rouge, LA 70803, USA: ${ }^{2}$ Department of \\ Pediatrics, Baylor College of Medicine, Children's Nutrition Research Center, Houston, TX, USA: ${ }^{3}$ NutraSource, \\ Clarksville, MD, USA: ${ }^{4}$ Department of Gastroenterology and Nutrition, Harvard Medical School, Boston, MA, USA
}

Submitted 10 March 2010: Accepted 14 July 2010: First published online 6 0ctober 2010

\begin{abstract}
Objective: To examine the association of consumption of whole grains (WG) with diet quality and nutrient intake in children and adolescents.

Design: Secondary analysis of cross-sectional data.

Setting: The 1999-2004 National Health and Nutrition Examination Survey.

Subjects: Children aged $2-5$ years ( $n$ 2278) and 6-12 years ( $n$ 3868) and adolescents aged 13-18 years ( $n$ 4931). The participants were divided into four WG consumption groups: $\geq 0$ to $<0 \cdot 6, \geq 0 \cdot 6$ to $<1 \cdot 5, \geq 1 \cdot 5$ to $<3 \cdot 0$ and $\geq 3 \cdot 0$ servings/d. Nutrient intake and diet quality, using the Healthy Eating Index (HEI)2005 , were determined for each group from a single $24 \mathrm{~h}$ dietary recall.

Results: The mean number of servings of WG consumed was $0.45,0.59$ and 0.63 for children/adolescents at the age of 2-5, 6-12 and 13-18 years, respectively. In all groups, HEI and intakes of energy, fibre, vitamin $\mathrm{B}_{6}$, folate, magnesium, phosphorus and iron were significantly higher in those consuming $\geq 3 \cdot 0$ servings of WG/d; intakes of protein, total fat, SFA and MUFA and cholesterol levels were lower. Intakes of PUFA ( $6-12$ years), vitamins $\mathrm{B}_{1}\left(2-5\right.$ and $13-18$ years), $\mathrm{B}_{2}$ (13-18 years), A ( $2-5$ and $13-18$ years) and E (13-18 years) were higher in those groups consuming $\geq 3 \cdot 0$ servings of $\mathrm{WG} / \mathrm{d}$; intakes of added sugars ( $2-5$ years), vitamin $\mathrm{C}$ ( $2-5$ and $6-12$ years), potassium and sodium (6-12 years) were lower.

Conclusions: Overall consumption of WG was low. Children and adolescents who consumed the most servings of WG had better diet quality and nutrient intake.
\end{abstract}

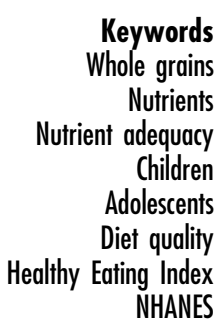

In the USA, the most commonly consumed grains are wheat, oats, rice, maize and barley, with wheat comprising $66-75 \%$ of the total ${ }^{(1,2)}$. Whole grains (WG) are defined as cereal grains that are intact, or ground or cracked fruit with the endosperm, germ and bran present in the same relative proportions as the intact grain ${ }^{(3,4)}$. Endosperm $(65-75 \%)$ is composed of starch, NSP and small amounts of protein and lipids. Germ (4-17\%) is a rich source of proteins, lipids, B vitamins and vitamin E. Bran contains dietary fibre; protein; B vitamins, including thiamin, niacin, riboflavin and pantothenic acid; and minerals, including calcium, magnesium and potassium ${ }^{(1,2,5,6)}$. WG also have other health-protective compounds, including flavonoids, lignans, resistant starches and phenols. During processing, the majority of the nutritive value of WG is preserved ${ }^{(2)}$.

Consumption of WG, unlike that of refined grains, is associated with a lower risk of CVD and stroke ${ }^{(7,8)}$, hypertension $^{(8,9)}$, type 2 diabetes $^{(10)}$ metabolic syndrome $^{(11)}$, obesity $^{(12)}$ and some cancers ${ }^{(13,14)}$. The mechanisms of these beneficial effects are not clear and the components of WG may act synergistically ${ }^{(1,15)}$. Most of these studies have been conducted in adults and little information is available for children or adolescents.

The 2005 Dietary Guidelines Advisory Committee recommended that at least half of the number of recommended grain servings be $\mathrm{WG}^{(16)}$. Thus, the WG recommendation for children varies; children as young as 2 years need only 1.5 servings (ounce equivalents), whereas children at the age of 9 years and above need 3 servings $/ \mathrm{d}^{(16,17)}$. These recommendations are supported by the American Academy of Pediatrics ${ }^{(18)}$.

Intake of WG in children and adolescents is not well documented, but studies conducted before the release of the 2005 Dietary Guidelines for Americans (DGA) 1989-1991 and 1994-1996 Continuing Survey of Food Intakes by Individuals (CSFII), it was shown that children and adolescents at the age of 2-19 years consumed a daily suggested that consumption was low. Using data from the 
average of 0.9 servings of WG in 1989-1991 and 1.0 serving in 1994-1996(19). In all, $7 \%$ and 9\% of children, respectively, consumed three servings of WG during the time frame of the study ${ }^{(19)}$. Low intake of WG was confirmed in another study using the CSFII data ${ }^{(20)}$ and with data from the 1999-2002 National Health and Nutrition Examination Survey (NHANES). Data from the NHANES showed that children and adolescents at the age of 6-19 years consumed only $0 \cdot 8-1 \cdot 0$ mean servings of $\mathrm{WG} / \mathrm{d}^{(21)}$. A smaller regional study of adolescents showed a slightly higher intake with female adolescents consuming 1.3 servings and male adolescents consuming $1 \cdot 4$ servings of $\mathrm{WG} / \mathrm{d}^{(11)}$.

Determining WG consumption has been difficult since the definition of WG has been unclear. The vast majority of studies $^{(22)}$ that have assessed WG intake were not based on the current definition of WG, but on the classification scheme proposed by Jacobs et al. ${ }^{(23)}$ in 1998, with WG defined as foods containing $\geq 25 \%$ WG or bran by weight. The current definition of WG was adopted by the Food and Drug Administration (FDA) in 2006 ${ }^{(4)}$. Currently, the FDA allows health claims for WG foods that contain $\geq 51 \% \mathrm{WG}$ ingredient(s) by weight per reference amount customarily consumed $^{(24)}$. The new FDA definition excludes bran and pearled barley as $\mathrm{WG}^{(24)}$. The US Department of Agriculture (USDA) MyPyramid Equivalents Database (MPED) versions $1^{(25)}$ and $2^{(26)}$ provides quantified measures of WG foods, and it provides information with and without bran (old and new definitions) respectively.

There are no recent studies using nationally representative data looking at the consumption of WG or the relationship of WG consumption with diet quality and nutrient intake of children and adolescents. The purpose of the present study was to examine the association of WG consumption, using the FDA definition of WG, with diet quality and nutrient intake in a recent, nationally representative sample of children and adolescents.

\section{Methods}

NHANES is a continuous programme that collects information about the nutrition and health status of the US population using a complex, multi-stage, stratified probability sample of the non-institutionalized civilian US population, aged 2 years and above ${ }^{(27)}$. As recommended, the NHANES data sets from 1999-2000, 2001-2002 and 2003-2004 were concatenated ${ }^{(28)}$.

\section{Dietary assessment method}

For data collection years, 1999-2002, a single multiple-pass $24 \mathrm{~h}$ dietary recall was conducted during an interview using computer-assisted software to record dietary intake data ${ }^{(29)}$. In 2003-2004, $2 \mathrm{~d}$ intakes were collected; however, for the present study, only the first day interview-administered recalls were used to assure consistency with the 1999-2002 dietary data. Parents of children 2-5 years of age provided the recalls; children (6-11 years) were assisted by an adult; and older children and adolescents (12-18 years) provided their own recall. Descriptions of these methods are provided in the NHANES Dietary Interviewer's Procedures Manual ${ }^{(30)}$.

\section{Participants and whole grains consumption categories}

NHANES data collected from 1999 to 2004 were used to compare diet quality and nutrient intake of children at the age of $2-5$ years ( $n$ 2278), 6-12 years ( $n$ 3868) and adolescents at the age of $13-18$ years $(n$ 4931). Pregnant and lactating female adolescents were excluded. In addition, there were six foods, principally breakfast cereals or bars, introduced in 2003 that could contain WG; however, there was no information available to calculate their WG content and the individuals ( $n$ 11) who consumed at least one of these products were also excluded. As this was a secondary data analysis with no personal identifiers, the present study was exempted by the Institutional Review Board of the LSU AgCenter.

The participants were categorized into one of four WG consumption categories: $\geq 0$ to $<0 \cdot 6, \geq 0 \cdot 6$ to $<1 \cdot 5, \geq 1 \cdot 5$ to $<3 \cdot 0$ and $\geq 3 \cdot 0$ servings $/$ d. This categorization was chosen since the recommendation for most children and adolescents is 3 servings/d; 1.5 servings represents onehalf of the recommendation; and the average number of servings was approximately $0 \cdot 6$ servings. WG intake was calculated using the new definition for WG (excluding bran) as outlined by the MPED ${ }^{(25,26)}$. The MPED food data files contain the number of MyPyramid equivalents/100 g of food by thirty-two MyPyramid food groups.

\section{Nutrient analysis}

The USDA 1994-98 Survey Nutrient Database ${ }^{(31)}$ was used to process the dietary interview data in NHANES 1999-2000, whereas the USDA Food and Nutrient Database for Dietary Studies (FNDDS), versions $1^{(32)}$ and $2^{(33)}$, were used in NHANES 2001-2002 and 2003-2004, respectively. In the original release of NHANES 19992000 , data on vitamin A intake were only available in $\mu \mathrm{g}$ retinol equivalents ( $\mu \mathrm{g} \mathrm{RE})$, vitamin $\mathrm{E}$ intake data were only available in $\mathrm{mg} \alpha$-tocopherol equivalents (mg ATE), only total folate $(\mu \mathrm{g})$ intake data, and no vitamin $\mathrm{K}(\mu \mathrm{g})$ or sugar (g) intake data were available as well. Currently, Dietary Reference Intakes (DRI) for vitamins A and E and folate are expressed as $\mu \mathrm{g}$ RAE, mg AT and dietary folate equivalents (DFE), respectively ${ }^{(34,35)}$. The special database released by the USDA to determine vitamin A as $\mu \mathrm{g}$ RAE and vitamin $\mathrm{E}$ as $\mathrm{mg}$ AT was used ${ }^{(36)}$. The FNDDS was used to append the intake of folate (DFE), vitamin $\mathrm{K}(\mu \mathrm{g})$ and total sugars (g) to the NHANES 1999-2000 database. The food composition data of added sugars were obtained from the MPED for USDA Survey Food Codes version $1 \cdot 0^{(25)}$.

The Healthy Eating Index (HEI)-2005 score was used to determine the diet quality ${ }^{(37)}$. The HEI contains twelve food 
components that reflect the recommendations of the DGA 2005. Dietary intake is expressed per $4184 \mathrm{~kJ}$ ( $1000 \mathrm{kcal}$ ) for all components except SFA and sodium, which are fixed recommendations. The maximum possible score on the index is 100 . The first six components (i.e. total fruit, whole fruit, total vegetable, dark green/orange vegetables and legumes, total grain and WG) are scored from 0 to 5 points. The next five components (i.e. milk, meat and beans, oil, SFA and sodium) are scored from 0 to 10 points; and the last component of solid fat, alcohol and added sugar is scored from 0 to 20 points. Scores were calculated proportionally, except for SFA and sodium; for these components, the scores were pro-rated linearly between 0-8 and $8-10$ points ( 8 and 10 points represented acceptable and optimal levels, respectively $)^{(37)}$. To calculate HEI, 'discretionary solid fat' and 'discretionary oil' were needed. The MPED (version 2) only provides 'Total Discretionary Fats' as a single group. To overcome this problem, a ratio of 'Discretionary Oil to Discretionary Solid Fat' was created for each food using the MPED (version 1), which had these fats separated. The SAS code used to calculate HEI scores was downloaded from the Center for Nutrition Policy and Promotion website ${ }^{(38)}$.

\section{Statistical analysis}

Sample-weighted data were used in all statistical analyses, and all analyses were performed using SUDAAN Release $9 \cdot 0 \cdot 1$ (Research Triangle Institute, Research Triangle Park, NC, USA) using a modified 6-year weight sample. A 6-year weight variable was created by assigning two-thirds of the 4-year weight for 1999-2002 if the person was sampled in 1999-2002 or assigning one-third of the 2-year weight for 2003-2004 if the person was sampled in 2003-2004. The unadjusted mean WG intake and counts and percentages of children and adolescents in WG consumption groups were calculated using SUDAAN. Nutrient intake was based on all foods consumed. Least-squares mean diet quality (HEI), total energy intake and macro- and micronutrient intakes were obtained by regressing intake variables on WG consumption groups. The models were adjusted for age, gender, ethnicity and total energy $(\mathrm{kJ}(\mathrm{kcal}))$. $P$ for trend was calculated using SUDAAN with WG intake as a linear independent variable in place of the WG categories. A probability of $\leq 0.05$ was considered significant.

\section{Results}

\section{Servings of the whole grains consumed by children and adolescents}

Table 1 shows the mean number of WG consumed by each of the three age groups. Children aged 2-5 years consumed an average of $0 \cdot 45$ servings of $\mathrm{WG} / \mathrm{d}$, whereas children aged 6-12 years and adolescents of 13-18 years of age consumed 0.59 and 0.63 servings, respectively. Only $1 \cdot 49 \%, 4 \cdot 00 \%$ and $4 \cdot 34 \%$ of children aged $2-5$,
Table 1 Mean number of servings of WG consumed and number (\%) of consumers for the three age groups by WG consumption group

\begin{tabular}{|c|c|c|c|c|c|c|}
\hline \multirow[b]{2}{*}{ WG servings } & \multicolumn{2}{|c|}{$\begin{array}{l}2-5 \text { years } \\
(n 2278)\end{array}$} & \multicolumn{2}{|c|}{$\begin{array}{c}\text { 6-12 years } \\
(n 3868)\end{array}$} & \multicolumn{2}{|c|}{$\begin{array}{c}\text { 13-18 years } \\
(n \text { 4931) }\end{array}$} \\
\hline & $n$ & $\%$ & $n$ & $\%$ & $n$ & $\%$ \\
\hline Mean & \multicolumn{2}{|c|}{0.45} & \multicolumn{2}{|c|}{0.59} & \multicolumn{2}{|c|}{0.63} \\
\hline$\geq 3$ & 34 & $1 \cdot 5$ & 153 & $4 \cdot 0$ & 214 & $4 \cdot 3$ \\
\hline$\geq 1.5$ to $<3.0$ & 156 & $6 \cdot 9$ & 328 & 8 . & 477 & $9 \cdot 7$ \\
\hline$\geq 0.6$ to $<1.5$ & 542 & $23 \cdot 8$ & 712 & $18 \cdot 4$ & 814 & $16 \cdot 5$ \\
\hline$\geq 0$ to $<0.6$ & 1546 & $67 \cdot 9$ & 2675 & $69 \cdot 2$ & 3426 & $69 \cdot 5$ \\
\hline
\end{tabular}

WG, whole grains.

6-12 years and adolescents aged 13-18 years, respectively, consumed $\geq 3$ servings of $\mathrm{WG} / \mathrm{d}$.

\section{Healtby Eating Index, energy and nutrient intakes for children aged 2-5 years}

HEI scores were significantly higher when more servings of WG were consumed $(P<0 \cdot 001 ;$ Table 2$)$. Intakes of energy $(P<0 \cdot 001)$, carbohydrates $(P<0 \cdot 001)$, fibre $(P<0 \cdot 001)$, vitamins A $(P=0 \cdot 05), \mathrm{B}_{1}(P=0 \cdot 04)$ and $\mathrm{B}_{6}$ $(P<0 \cdot 001)$, folate $(P<0 \cdot 001)$, magnesium $(P<0 \cdot 001)$, phosphorus $(P=0 \cdot 0015)$ and iron $(P<0 \cdot 001)$ were significantly higher when an increased number of servings of WG was consumed. Intakes of protein $(P=0.05)$, added sugars $(P=0 \cdot 04)$, total fat $(P=0 \cdot 01)$, SFA $(P<$ $0 \cdot 001)$, MUFA $(P<0 \cdot 001)$, cholesterol $(P<0 \cdot 001)$ and vitamin $\mathrm{C}(P=0 \cdot 01)$ were significantly lower when an increased number of servings of WG was consumed.

\section{Healtby Eating Index, energy and nutrient intakes for children aged 6-12 years}

HEI scores were significantly higher when an increased number of servings of WG was consumed $(P<0 \cdot 001$; Table 3). Energy $(P<0 \cdot 001)$, carbohydrates $(P<0 \cdot 001)$, fibre $(P<0 \cdot 001)$, PUFA $(P=0 \cdot 01)$, vitamin $\mathrm{B}_{6}(P=0 \cdot 0037)$, folate $(P=0 \cdot 04)$, magnesium $(P<0 \cdot 001)$, phosphorus $(P<0 \cdot 001)$ and iron $(P<0 \cdot 001)$ intakes were higher when more servings of WG were consumed. Intakes of protein $(P=0 \cdot 01)$, total fat $(P=0 \cdot 05)$, SFA $(P<0 \cdot 001)$, MUFA $(P=0 \cdot 01)$, cholesterol $(P<0 \cdot 001)$, vitamin $\mathrm{C}(P<0 \cdot 001)$, potassium $(P=0 \cdot 05)$ and sodium $(P=0.05)$ were lower when more servings of WG were consumed.

\section{Healtby Eating Index, energy and nutrient intakes for adolescents aged 13-18 years}

HEI scores were significantly higher when more servings of WG were consumed $(P<0 \cdot 001$; Table 4). Energy $(P<0 \cdot 001)$, carbohydrates $(P<0 \cdot 001)$, fibre $(P<0 \cdot 001)$, vitamins A $(P=0.04), \mathrm{E}(P=0.03), \mathrm{B}_{1}(P=0.03), \mathrm{B}_{2}$ $(P<0 \cdot 001)$ and $\mathrm{B}_{6}(P<0 \cdot 001)$, folate $(P<0 \cdot 001)$, magnesium $(P<0.001)$, phosphorus $(P=0.03)$ and iron $(P<0 \cdot 001)$ intakes were significantly higher when more servings of $\mathrm{WG}$ were consumed. Intakes of protein $(P<0 \cdot 001)$, total fat $(P<0 \cdot 001)$, SFA $(P<0 \cdot 001)$, MUFA 
Table 2 Diet quality, daily total energy and nutrient intakes by WG consumption groups, US children aged 2-5 years, NHANES 1999-2004*

\begin{tabular}{|c|c|c|c|c|c|c|c|c|c|c|c|}
\hline & \multirow{2}{*}{\multicolumn{2}{|c|}{$\begin{array}{l}\text { Total population } \\
\quad(n 2278)\end{array}$}} & \multicolumn{8}{|c|}{ WG consumption group (servings/d) } & \multirow[b]{3}{*}{$P$ for trend } \\
\hline & & & \multicolumn{2}{|c|}{$\begin{array}{l}\geq 0 \text { to }<0 \cdot 6 \\
(n 1546)\end{array}$} & \multicolumn{2}{|c|}{$\begin{array}{c}\geq 0 \cdot 6 \text { to }<1.5 \\
(n 542)\end{array}$} & \multicolumn{2}{|c|}{$\begin{array}{c}\geq 1 \cdot 5 \text { to }<3 \cdot 0 \\
(n 156)\end{array}$} & \multicolumn{2}{|c|}{$\begin{array}{c}\geq 3 \\
(n 34)\end{array}$} & \\
\hline & Mean & SE & Mean & SE & Mean & SE & Mean & SE & Mean & SE & \\
\hline WG servingst & 0.48 & 0.02 & $0 \cdot 14$ & 0.01 & 0.96 & 0.02 & 1.97 & 0.04 & $3 \cdot 82$ & $0 \cdot 20$ & $<0.001$ \\
\hline $\mathrm{HEI}$ & $52 \cdot 98$ & 0.33 & $51 \cdot 69$ & 0.38 & $56 \cdot 02$ & 0.77 & $57 \cdot 41$ & $1 \cdot 30$ & $57 \cdot 50$ & $2 \cdot 46$ & $<0.001$ \\
\hline Energy (kcal) & $1659 \cdot 56$ & $15 \cdot 24$ & $1621 \cdot 10$ & $17 \cdot 87$ & $1730 \cdot 09$ & $37 \cdot 18$ & $1806 \cdot 80$ & $53 \cdot 30$ & $1945 \cdot 19$ & $85 \cdot 22$ & $<0.001$ \\
\hline Energy (kJ) & $6948 \cdot 25$ & $63 \cdot 81$ & $6787 \cdot 22$ & $74 \cdot 82$ & $7243 \cdot 54$ & $155 \cdot 67$ & $7564 \cdot 71$ & $223 \cdot 16$ & $8144 \cdot 12$ & $356 \cdot 8$ & $<0.001$ \\
\hline Protein (g) & $56 \cdot 37$ & 0.38 & $56 \cdot 57$ & 0.45 & $56 \cdot 10$ & $0 \cdot 86$ & $55 \cdot 97$ & $1 \cdot 90$ & $52 \cdot 51$ & $2 \cdot 11$ & 0.05 \\
\hline Carbohydrate (g) & $230 \cdot 49$ & $1 \cdot 05$ & $228 \cdot 18$ & $1 \cdot 23$ & $235 \cdot 53$ & $2 \cdot 39$ & $237 \cdot 18$ & $5 \cdot 21$ & $247 \cdot 67$ & $9 \cdot 19$ & $<0.001$ \\
\hline Fibre (g) & $10 \cdot 65$ & $0 \cdot 11$ & $9 \cdot 91$ & $0 \cdot 12$ & 11.94 & $0 \cdot 27$ & $13 \cdot 79$ & 0.51 & $15 \cdot 79$ & $0 \cdot 79$ & $<0.001$ \\
\hline Total sugars (g) & $124 \cdot 65$ & 0.99 & $125 \cdot 29$ & $1 \cdot 19$ & $124 \cdot 38$ & $2 \cdot 30$ & $121 \cdot 38$ & $4 \cdot 21$ & $113 \cdot 98$ & $9 \cdot 49$ & 0.24 \\
\hline Added sugars (g) & $16 \cdot 06$ & 0.25 & $16 \cdot 33$ & 0.30 & $15 \cdot 94$ & 0.59 & $13 \cdot 77$ & $1 \cdot 20$ & $14 \cdot 96$ & $2 \cdot 36$ & 0.04 \\
\hline Total fat $(\mathrm{g})$ & $59 \cdot 42$ & $0 \cdot 38$ & $60 \cdot 18$ & 0.44 & $57 \cdot 61$ & $0 \cdot 88$ & $57 \cdot 41$ & $1 \cdot 90$ & $54 \cdot 58$ & 3.93 & 0.01 \\
\hline SFA $(g)$ & $22 \cdot 04$ & $0 \cdot 19$ & $22 \cdot 41$ & 0.22 & $21 \cdot 43$ & 0.44 & $20 \cdot 57$ & $0 \cdot 82$ & $18 \cdot 62$ & $1 \cdot 83$ & $<0.001$ \\
\hline MUFA (g) & $22 \cdot 10$ & $0 \cdot 17$ & $22 \cdot 52$ & $0 \cdot 20$ & $21 \cdot 13$ & 0.38 & $20 \cdot 88$ & 0.72 & $19 \cdot 66$ & $1 \cdot 65$ & $<0.001$ \\
\hline PUFA (g) & $10 \cdot 53$ & $0 \cdot 11$ & $10 \cdot 46$ & $0 \cdot 13$ & $10 \cdot 35$ & 0.25 & $11 \cdot 43$ & 0.53 & $12 \cdot 08$ & $1 \cdot 24$ & $0 \cdot 14$ \\
\hline Cholesterol (mg) & $185 \cdot 70$ & $3 \cdot 25$ & $191 \cdot 68$ & $3 \cdot 70$ & $173 \cdot 63$ & $8 \cdot 10$ & $160 \cdot 34$ & $15 \cdot 57$ & $162 \cdot 01$ & $27 \cdot 51$ & $<0.001$ \\
\hline Vitamin A ( $\mu \mathrm{g}$ RAE) & $605 \cdot 14$ & $13 \cdot 62$ & $592 \cdot 77$ & $16 \cdot 29$ & $626 \cdot 24$ & $32 \cdot 76$ & $679 \cdot 05$ & $58 \cdot 59$ & $612 \cdot 70$ & $103 \cdot 04$ & 0.05 \\
\hline Vitamin C (mg) & $96 \cdot 04$ & $2 \cdot 08$ & $98 \cdot 22$ & $2 \cdot 52$ & $92 \cdot 80$ & $5 \cdot 04$ & $84 \cdot 38$ & $6 \cdot 43$ & $84 \cdot 52$ & $16 \cdot 15$ & 0.01 \\
\hline Vitamin E (mg AT) & $4 \cdot 79$ & 0.08 & $4 \cdot 72$ & 0.09 & $5 \cdot 18$ & $0 \cdot 27$ & $4 \cdot 66$ & $0 \cdot 19$ & $4 \cdot 34$ & $0 \cdot 41$ & $0 \cdot 78$ \\
\hline Vitamin $B_{1}(\mathrm{mg})$ & $1 \cdot 33$ & 0.01 & $1 \cdot 31$ & 0.01 & $1 \cdot 41$ & 0.03 & $1 \cdot 33$ & 0.04 & $1 \cdot 45$ & 0.13 & 0.04 \\
\hline Vitamin $B_{2}(\mathrm{mg})$ & 1.98 & 0.02 & 1.96 & 0.02 & $2 \cdot 04$ & 0.04 & $2 \cdot 02$ & 0.08 & 1.96 & $0 \cdot 16$ & $0 \cdot 18$ \\
\hline Vitamin $\mathrm{B}_{6}(\mathrm{mg})$ & $1 \cdot 44$ & 0.02 & $1 \cdot 40$ & 0.02 & 1.53 & 0.04 & $1 \cdot 59$ & 0.07 & $1 \cdot 67$ & $0 \cdot 21$ & $<0.001$ \\
\hline Vitamin $B_{12}(\mu \mathrm{g})$ & $4 \cdot 28$ & 0.08 & $4 \cdot 29$ & $0 \cdot 10$ & $4 \cdot 41$ & 0.24 & $4 \cdot 08$ & $0 \cdot 28$ & $3 \cdot 20$ & $0 \cdot 41$ & 0.07 \\
\hline Niacin (mg) & $15 \cdot 84$ & $0 \cdot 16$ & $15 \cdot 60$ & $0 \cdot 17$ & $16 \cdot 50$ & 0.37 & $16 \cdot 08$ & 0.72 & $18 \cdot 26$ & $2 \cdot 51$ & 0.09 \\
\hline Folate $(\mu \mathrm{g})$ & $317 \cdot 38$ & $4 \cdot 19$ & $305 \cdot 64$ & $4 \cdot 42$ & $350 \cdot 97$ & $10 \cdot 78$ & $342 \cdot 94$ & $25 \cdot 55$ & $354 \cdot 76$ & $38 \cdot 58$ & $<0.001$ \\
\hline Calcium (mg) & $929 \cdot 73$ & $11 \cdot 08$ & $923 \cdot 21$ & $13 \cdot 41$ & $941 \cdot 03$ & $23 \cdot 88$ & $991 \cdot 23$ & $47 \cdot 54$ & $846 \cdot 78$ & $66 \cdot 24$ & 0.23 \\
\hline Magnesium (mg) & $200 \cdot 92$ & $1 \cdot 41$ & $192 \cdot 32$ & $1 \cdot 55$ & $214 \cdot 14$ & 3.06 & $240 \cdot 41$ & $6 \cdot 80$ & $266 \cdot 01$ & $13 \cdot 96$ & $<0.001$ \\
\hline Potassium (mg) & $2128 \cdot 61$ & $16 \cdot 17$ & $2130 \cdot 98$ & $19 \cdot 21$ & $2119 \cdot 29$ & $36 \cdot 31$ & $2212 \cdot 44$ & $72 \cdot 69$ & $1811 \cdot 32$ & $110 \cdot 47$ & 0.35 \\
\hline Phosphorus (mg) & $1091 \cdot 00$ & $8 \cdot 31$ & $1075 \cdot 74$ & $9 \cdot 85$ & $1119 \cdot 00$ & $18 \cdot 27$ & $1173 \cdot 63$ & $40 \cdot 12$ & $1112 \cdot 58$ & $44 \cdot 07$ & $0 \cdot 0015$ \\
\hline Iron (mg) & $12 \cdot 38$ & $0 \cdot 14$ & $11 \cdot 69$ & $0 \cdot 13$ & $13 \cdot 72$ & 0.37 & $14 \cdot 62$ & $0 \cdot 83$ & $18 \cdot 04$ & $2 \cdot 62$ & $<0.001$ \\
\hline Sodium (mg) & $2457 \cdot 35$ & $19 \cdot 15$ & $2471 \cdot 26$ & $23 \cdot 05$ & $2452 \cdot 54$ & $45 \cdot 03$ & $2400 \cdot 94$ & $76 \cdot 13$ & $2155 \cdot 36$ & $123 \cdot 03$ & 0.06 \\
\hline
\end{tabular}

WG, whole grains; NHANES, National Health and Nutrition Examination Survey; HEI, Healthy Eating Index; RAE, retinol activity equivalents; AT, $\alpha$-tocopherol. *Estimates were adjusted for age, gender, ethnicity and total energy intake. Models for total energy did not include total energy intake. tWG was defined according to the new definition that excludes bran.

$(P=0 \cdot 05)$ and cholesterol $(P<0 \cdot 001)$ were significantly lower when more servings of WG were consumed.

\section{Discussion}

The present study showed that although the overall consumption of WG was low, increasing consumption of WG was associated with improved diet quality and nutrient intake in children and adolescents. The number of servings increased slightly in each of the age groups, suggesting that older children and adolescents were more likely to consume WG than younger ones. In children aged 2-5 years, only $1.5 \%$ consumed three or more servings of WG/d; in this age group the consumption of 1.5-2.99 servings, which more closely matches the recommendations ${ }^{(17)}$, was approximately $7 \%$. The number of WG servings consumed by children and adolescents in the present study was lower than previously reported ${ }^{(19-21)}$, but this is likely the result of the definition of WG used. When the current definition of WG was used ${ }^{(26,27)}$, there were fewer servings consumed than when the older definition was used (data not shown).
The low consumption of WG by adults is a reason for children not consuming $\mathrm{WG}^{(8,12,39-41)}$. Interestingly, adults living with children in the household consumed fewer servings of WG than those who did not ${ }^{(40)}$. Thus, WG foods are not available to children. Parents influence what children eat by determining home food availability and accessibility ${ }^{(42-44)}$; parenting style or practices ${ }^{(45,46)}$, role modelling ${ }^{(44,47,48)}$ and the level to which they are influenced by their children's food preferences ${ }^{(49)}$ affect what foods are consumed. Adolescents show increasing control over their food choices ${ }^{(50)}$. For adolescents, some ${ }^{(50)}$, but not all studies ${ }^{(44,51)}$ suggest home availability and parenting style are less important than in younger children. Taste and personal health beliefs are important influences on an adolescent's choice of foods ${ }^{(51)}$. Influences on consumption of WG in children and adolescents are understudied ${ }^{(47,49)}$.

Little is known about barriers to WG consumption in children, but taste, texture and appearance were important in one study, with children preferring refined, sweetened grain products ${ }^{(49)}$. Younger children did not cite health as a reason for consuming WG and they were unable to identify WG foods, whereas older children could identify them and relate some of their health benefits ${ }^{(49)}$. 
Table 3 Diet quality, daily total energy and nutrient intakes by WG consumption groups, US children aged 6-12 years, NHANES 1999-2004*

\begin{tabular}{|c|c|c|c|c|c|c|c|c|c|c|c|}
\hline & \multirow{2}{*}{\multicolumn{2}{|c|}{$\begin{array}{l}\text { Total population } \\
\quad(n 3868)\end{array}$}} & \multicolumn{8}{|c|}{ WG consumption group (servings/d) } & \multirow[b]{3}{*}{$P$ for trend } \\
\hline & & & \multicolumn{2}{|c|}{$\begin{array}{c}\geq 0 \text { to }<0 \cdot 6 \\
(n 2675)\end{array}$} & \multicolumn{2}{|c|}{$\begin{array}{l}\geq 0 \cdot 6 \text { to }<1.5 \\
\quad(n 712)\end{array}$} & \multicolumn{2}{|c|}{$\begin{array}{l}\geq 1 \cdot 5 \text { to }<3 \cdot 0 \\
\quad(n 328)\end{array}$} & \multicolumn{2}{|c|}{$\begin{array}{c}\geq 3 \\
(n 153)\end{array}$} & \\
\hline & Mean & SE & Mean & SE & Mean & SE & Mean & SE & Mean & SE & \\
\hline WG servingst & 0.65 & 0.02 & $0 \cdot 12$ & 0.01 & 0.99 & 0.01 & $2 \cdot 02$ & 0.03 & $4 \cdot 31$ & $0 \cdot 12$ & \\
\hline $\mathrm{HEI}$ & $49 \cdot 33$ & 0.29 & $47 \cdot 64$ & 0.35 & $51 \cdot 61$ & 0.66 & $53 \cdot 89$ & 0.99 & $56 \cdot 23$ & $1 \cdot 31$ & $<0.001$ \\
\hline Energy (kcal) & $2041 \cdot 36$ & $17 \cdot 52$ & $1955 \cdot 30$ & $22 \cdot 07$ & $2124 \cdot 35$ & 38.58 & $2259 \cdot 31$ & $50 \cdot 47$ & $2546 \cdot 66$ & $77 \cdot 53$ & $<0.001$ \\
\hline Energy (kJ) & $8546 \cdot 77$ & $73 \cdot 35$ & $8186 \cdot 45$ & $92 \cdot 40$ & $8894 \cdot 23$ & $161 \cdot 53$ & $9459 \cdot 28$ & $211 \cdot 31$ & $10662 \cdot 36$ & $324 \cdot 60$ & $<0.001$ \\
\hline Protein $(\mathrm{g})$ & $68 \cdot 02$ & 0.44 & $68 \cdot 88$ & 0.55 & $67 \cdot 54$ & $1 \cdot 18$ & $64 \cdot 05$ & $1 \cdot 46$ & $65 \cdot 04$ & $2 \cdot 32$ & $0 \cdot 01$ \\
\hline Carbohydrate $(\mathrm{g})$ & $280 \cdot 42$ & 1.04 & $277 \cdot 00$ & $1 \cdot 26$ & $284 \cdot 66$ & $2 \cdot 57$ & 291.97 & 3.34 & $291 \cdot 44$ & $6 \cdot 24$ & $<0.001$ \\
\hline Fibre & $12 \cdot 60$ & $0 \cdot 12$ & $11 \cdot 77$ & $0 \cdot 14$ & $13 \cdot 03$ & 0.25 & $15 \cdot 18$ & 0.47 & $17 \cdot 98$ & 0.63 & $<0.001$ \\
\hline Total sugars & $144 \cdot 62$ & $1 \cdot 09$ & $144 \cdot 98$ & $1 \cdot 37$ & $144 \cdot 85$ & $2 \cdot 46$ & $148 \cdot 67$ & $3 \cdot 68$ & $130 \cdot 88$ & $6 \cdot 29$ & $0 \cdot 16$ \\
\hline Added sugars & 23.97 & $0 \cdot 28$ & $24 \cdot 15$ & 0.34 & $23 \cdot 82$ & 0.65 & $24 \cdot 48$ & $1 \cdot 06$ & $21 \cdot 00$ & $1 \cdot 61$ & $0 \cdot 17$ \\
\hline Total fat $(\mathrm{g})$ & $74 \cdot 77$ & 0.38 & $75 \cdot 58$ & 0.48 & $73 \cdot 33$ & $0 \cdot 88$ & $72 \cdot 55$ & $1 \cdot 23$ & $72 \cdot 87$ & $2 \cdot 41$ & 0.05 \\
\hline SFA (g) & $26 \cdot 55$ & $0 \cdot 18$ & $27 \cdot 18$ & 0.21 & $25 \cdot 53$ & 0.44 & $25 \cdot 27$ & 0.55 & $23 \cdot 72$ & $1 \cdot 08$ & $<0.001$ \\
\hline MUFA (g) & $28 \cdot 28$ & $0 \cdot 17$ & $28 \cdot 65$ & 0.22 & $28 \cdot 00$ & 0.38 & $27 \cdot 06$ & 0.51 & $26 \cdot 41$ & $1 \cdot 05$ & 0.01 \\
\hline PUFA (g) & $14 \cdot 15$ & $0 \cdot 14$ & $13 \cdot 91$ & $0 \cdot 18$ & $14 \cdot 02$ & $0 \cdot 30$ & $14 \cdot 66$ & 0.56 & $17 \cdot 22$ & 0.91 & 0.01 \\
\hline Cholesterol (mg) & $220 \cdot 97$ & $3 \cdot 51$ & $235 \cdot 00$ & $4 \cdot 88$ & $202 \cdot 08$ & $5 \cdot 36$ & $177 \cdot 58$ & $11 \cdot 08$ & $173 \cdot 72$ & $14 \cdot 97$ & $<0.001$ \\
\hline Vitamin A ( $\mu \mathrm{g}$ RAE) & $660 \cdot 90$ & $11 \cdot 56$ & $662 \cdot 69$ & $14 \cdot 19$ & $641 \cdot 53$ & $23 \cdot 47$ & $666 \cdot 19$ & $36 \cdot 40$ & $698 \cdot 42$ & $76 \cdot 82$ & 0.56 \\
\hline Vitamin C (mg) & $82 \cdot 60$ & $1 \cdot 65$ & $84 \cdot 23$ & $2 \cdot 05$ & $83 \cdot 56$ & 3.90 & $81 \cdot 70$ & $6 \cdot 83$ & $57 \cdot 40$ & $5 \cdot 42$ & $<0.001$ \\
\hline Vitamin $E$ (mg AT) & $6 \cdot 15$ & 0.08 & $6 \cdot 06$ & 0.09 & $6 \cdot 09$ & 0.19 & $6 \cdot 73$ & 0.41 & $6 \cdot 64$ & 0.43 & $0 \cdot 14$ \\
\hline Vitamin $B_{1}(\mathrm{mg})$ & $1 \cdot 60$ & 0.01 & $1 \cdot 57$ & 0.01 & $1 \cdot 64$ & 0.03 & $1 \cdot 66$ & 0.06 & $1 \cdot 64$ & 0.08 & $0 \cdot 10$ \\
\hline Vitamin $B_{2}(\mathrm{mg})$ & $2 \cdot 13$ & 0.02 & $2 \cdot 10$ & 0.02 & $2 \cdot 21$ & 0.05 & $2 \cdot 23$ & 0.06 & $2 \cdot 21$ & $0 \cdot 11$ & 0.07 \\
\hline Vitamin $B_{6}(\mathrm{mg})$ & $1 \cdot 62$ & 0.02 & $1 \cdot 57$ & 0.02 & $1 \cdot 71$ & 0.04 & $1 \cdot 72$ & 0.07 & $1 \cdot 78$ & $0 \cdot 10$ & 0.004 \\
\hline Vitamin $B_{12}(\mu \mathrm{g})$ & $4 \cdot 70$ & 0.08 & $4 \cdot 75$ & $0 \cdot 11$ & $4 \cdot 67$ & 0.14 & $4 \cdot 64$ & 0.32 & $4 \cdot 20$ & 0.34 & $0 \cdot 18$ \\
\hline Niacin (mg) & $20 \cdot 03$ & $0 \cdot 17$ & $19 \cdot 86$ & $0 \cdot 20$ & $20 \cdot 71$ & 0.42 & $19 \cdot 83$ & 0.68 & $20 \cdot 26$ & $1 \cdot 01$ & 0.42 \\
\hline Folate $(\mu \mathrm{g})$ & 376.09 & $4 \cdot 96$ & $361 \cdot 02$ & $4 \cdot 21$ & $402 \cdot 26$ & $15 \cdot 12$ & $423 \cdot 12$ & $23 \cdot 80$ & $403 \cdot 72$ & $45 \cdot 77$ & 0.04 \\
\hline Calcium (mg) & $957 \cdot 29$ & $9 \cdot 86$ & $950 \cdot 69$ & $11 \cdot 72$ & $967 \cdot 00$ & $28 \cdot 19$ & $1004 \cdot 49$ & $34 \cdot 05$ & $925 \cdot 92$ & $55 \cdot 01$ & 0.91 \\
\hline Magnesium (mg) & $223 \cdot 23$ & $1 \cdot 49$ & $212 \cdot 24$ & $1 \cdot 70$ & $230 \cdot 28$ & 3.52 & $257 \cdot 15$ & $5 \cdot 79$ & $289 \cdot 64$ & $8 \cdot 52$ & $<0.001$ \\
\hline Potassium (mg) & $2211 \cdot 77$ & $16 \cdot 79$ & $2233 \cdot 41$ & $21 \cdot 45$ & $2175 \cdot 33$ & $40 \cdot 60$ & $2183 \cdot 95$ & $56 \cdot 97$ & $2092 \cdot 61$ & $86 \cdot 34$ & 0.05 \\
\hline Phosphorus (mg) & $1222 \cdot 74$ & $8 \cdot 22$ & $1200 \cdot 19$ & $10 \cdot 26$ & $1239 \cdot 17$ & $21 \cdot 85$ & $1302 \cdot 35$ & $27 \cdot 30$ & $1332 \cdot 69$ & $48 \cdot 59$ & $<0.001$ \\
\hline Iron (mg) & $14 \cdot 80$ & $0 \cdot 14$ & $14 \cdot 03$ & $0 \cdot 13$ & $15 \cdot 68$ & 0.34 & $16 \cdot 78$ & 0.66 & $18 \cdot 73$ & $1 \cdot 26$ & $<0.001$ \\
\hline Sodium (mg) & $3148 \cdot 04$ & $21 \cdot 49$ & 3188.07 & $28 \cdot 50$ & $3121 \cdot 01$ & $51 \cdot 59$ & $2925 \cdot 36$ & $72 \cdot 80$ & $3098 \cdot 33$ & $105 \cdot 98$ & 0.05 \\
\hline
\end{tabular}

WG, whole grains; NHANES, National Health and Nutrition Examination Survey; HEI, Healthy Eating Index; RAE, retinol activity equivalents; AT, $\alpha$-tocopherol. *Estimates were adjusted for age, gender, ethnicity and total energy intake. Models for total energy did not include total energy intake. +WG were defined according to the new definition that excludes bran.

The eating habits developed in childhood can track into adulthood ${ }^{(52-54)}$; thus, it is important to encourage healthy eating habits early in life. Recently, it was shown that a school-based intervention could increase WG consumption by one serving and decrease refined grain consumption by one serving in fourth and fifth grade students ${ }^{(55)}$. School is an appropriate venue for improving WG consumption in children. This is important, since few WG were served at school ${ }^{(19)}$. Although the USDA has issued a policy encouraging adherence to the DGA in the National School Lunch Program ${ }^{(56)}$, the law requires only that sponsors must offer grains, which can be either enriched or $\mathrm{WG}^{(57)}$. An Institute of Medicine Report ${ }^{(58)}$ has called for industry and schools to work together to increase availability of WG in child nutrition programmes.

Consumption of WG improved diet quality as indicated by the increasing HEI scores ${ }^{(37,59)}$ across the WG consumption groups. The HEI-2005 is designed to reflect the 2005 DGA $^{(37)}$. That version of the HEI includes a WG component, and includes energy from solid fat, alcohol and added sugars. Inclusion of the last three components should assuage concerns about the use of the HEI with children $^{(59)}$. The HEI scores are presented as the total score, which is appropriate for studying populations ${ }^{(37)}$. Although HEI scores improved with increasing levels of WG consumption, the mean score of those consuming $\geq 3$ servings of $\mathrm{WG} / \mathrm{d}$ ranged from 54 to 58 out of a maximum of 100 in the different age groups suggesting that overall diet quality of both children and adolescents could be improved ${ }^{(60)}$. The impact of $\mathrm{WG}$ may be small because of the low consumption levels.

Intakes of many macro- and micronutrients also improved with increased consumption of WG. The focus of this discussion will be on the shortfall nutrients in the diets of children (fibre, magnesium, vitamin E, calcium and potassium) ${ }^{(16)}$. These shortfall nutrients are found naturally in $\mathrm{WG}^{(1)}$.

The majority of children do not meet the daily fibre requirement, with mean intake approximately half of the recommendation $^{(61)}$ for Adequate Intake ${ }^{(62)}$. The present study showed that increased WG consumption was associated with increased fibre intake; however, mean intake fell below recommendations for all age groups. In children and adolescents, fibre intake is inversely associated with serum cholesterol levels ${ }^{(63)}$ and constipation ${ }^{(64)}$, which is a major cause of morbidity in children ${ }^{(65)}$. Fruit, vegetable and WG 
Table 4 Diet quality, daily total energy and nutrient intakes by WG consumption groups, US adolescents aged 13-18 years, NHANES 1999-2004*

\begin{tabular}{|c|c|c|c|c|c|c|c|c|c|c|c|}
\hline & \multirow{2}{*}{\multicolumn{2}{|c|}{$\begin{array}{l}\text { Total population } \\
\quad(n 4931)\end{array}$}} & \multicolumn{8}{|c|}{ WG consumption group (servings/d) } & \multirow[b]{3}{*}{$P$ for trenc } \\
\hline & & & \multicolumn{2}{|c|}{$\begin{array}{c}\geq 0 \text { to }<0 \cdot 6 \\
(n 3426)\end{array}$} & \multicolumn{2}{|c|}{$\begin{array}{c}\geq 0.6 \text { to }<1.5 \\
(n 814)\end{array}$} & \multicolumn{2}{|c|}{$\begin{array}{c}\geq 1.5 \text { to }<3.0 \\
(n 477)\end{array}$} & \multicolumn{2}{|c|}{$\begin{array}{c}\geq 3 \\
(n 214)\end{array}$} & \\
\hline & Mean & SE & Mean & SE & Mean & SE & Mean & SE & Mean & SE & \\
\hline WG servingst & 0.68 & 0.02 & 0.09 & 0.01 & 0.99 & 0.01 & $2 \cdot 04$ & 0.03 & $4 \cdot 73$ & $0 \cdot 17$ & \\
\hline $\mathrm{HEI}$ & $47 \cdot 68$ & 0.25 & $45 \cdot 54$ & 0.29 & $50 \cdot 22$ & 0.59 & $54 \cdot 02$ & 0.77 & $54 \cdot 36$ & 0.96 & $<0.001$ \\
\hline Energy (kcal) & $2340 \cdot 68$ & $20 \cdot 74$ & $2214 \cdot 49$ & $24 \cdot 41$ & $2380 \cdot 09$ & $43 \cdot 59$ & $2719 \cdot 68$ & 74.53 & $3118 \cdot 41$ & $116 \cdot 37$ & $<0.001$ \\
\hline Energy $(\mathrm{kJ})$ & $9799 \cdot 96$ & $86 \cdot 83$ & $9271 \cdot 63$ & $102 \cdot 20$ & $9964 \cdot 96$ & $182 \cdot 50$ & $11386 \cdot 76$ & $312 \cdot 04$ & $13056 \cdot 16$ & $487 \cdot 22$ & $<0.001$ \\
\hline Protein $(\mathrm{g})$ & 80.57 & 0.51 & $81 \cdot 20$ & 0.63 & $81 \cdot 86$ & $1 \cdot 34$ & 78.58 & 1.66 & 71.57 & $2 \cdot 89$ & $<0.001$ \\
\hline Carbohydrate (g) & $314 \cdot 79$ & $1 \cdot 21$ & $311 \cdot 51$ & $1 \cdot 60$ & $312 \cdot 40$ & $2 \cdot 66$ & $328 \cdot 56$ & 3.68 & 338.90 & $6 \cdot 14$ & $<0.001$ \\
\hline Fibre (g) & $13 \cdot 61$ & 0.13 & $12 \cdot 38$ & $0 \cdot 14$ & $14 \cdot 12$ & 0.27 & $16 \cdot 86$ & 0.46 & $21 \cdot 81$ & 0.76 & $<0.001$ \\
\hline Total sugars $(\mathrm{g})$ & $164 \cdot 87$ & $1 \cdot 33$ & $165 \cdot 90$ & $1 \cdot 83$ & $162 \cdot 92$ & $2 \cdot 80$ & $166 \cdot 86$ & 3.72 & $153 \cdot 51$ & $6 \cdot 29$ & $0 \cdot 16$ \\
\hline Added sugars (g) & $29 \cdot 71$ & 0.34 & $30 \cdot 15$ & 0.47 & $29 \cdot 18$ & 0.73 & 28.97 & 1.02 & $27 \cdot 15$ & 1.67 & 0.11 \\
\hline Total fat $(\mathrm{g})$ & $85 \cdot 58$ & 0.43 & $86 \cdot 16$ & 0.54 & $86 \cdot 82$ & 0.94 & $82 \cdot 32$ & $1 \cdot 43$ & $80 \cdot 12$ & $2 \cdot 60$ & $<0.001$ \\
\hline SFA (g) & $29 \cdot 74$ & $0 \cdot 18$ & $30 \cdot 26$ & 0.23 & $29 \cdot 96$ & 0.39 & $27 \cdot 99$ & 0.58 & $25 \cdot 58$ & $1 \cdot 15$ & $<0.001$ \\
\hline MUFA (g) & $32 \cdot 52$ & 0.18 & $32 \cdot 70$ & 0.23 & $33 \cdot 31$ & 0.43 & $30 \cdot 95$ & 0.58 & $30 \cdot 60$ & 1.08 & 0.05 \\
\hline PUFA (g) & $16 \cdot 62$ & $0 \cdot 18$ & $16 \cdot 44$ & 0.22 & $16 \cdot 81$ & $0 \cdot 40$ & $16 \cdot 94$ & 0.70 & $17 \cdot 64$ & 0.87 & $0 \cdot 21$ \\
\hline Cholesterol (mg) & $258 \cdot 60$ & 3.75 & $266 \cdot 84$ & 4.91 & $266 \cdot 50$ & 8.95 & 223.62 & $10 \cdot 29$ & $192 \cdot 07$ & $19 \cdot 29$ & $<0.001$ \\
\hline Vitamin A ( $\mu \mathrm{g}$ RAE) & $646 \cdot 90$ & $13 \cdot 96$ & $618 \cdot 69$ & $18 \cdot 39$ & $668 \cdot 33$ & $29 \cdot 69$ & $742 \cdot 10$ & $45 \cdot 47$ & $753 \cdot 26$ & $59 \cdot 42$ & 0.04 \\
\hline Vitamin $\mathrm{C}(\mathrm{mg})$ & $91 \cdot 85$ & 1.91 & $90 \cdot 92$ & $2 \cdot 48$ & $90 \cdot 25$ & 3.97 & $102 \cdot 40$ & $6 \cdot 85$ & $87 \cdot 76$ & $9 \cdot 81$ & 0.73 \\
\hline Vitamin E (mg AT) & $7 \cdot 14$ & 0.12 & $6 \cdot 89$ & $0 \cdot 12$ & $7 \cdot 13$ & 0.22 & $8 \cdot 58$ & 0.70 & $7 \cdot 49$ & 0.49 & 0.03 \\
\hline Vitamin $B_{1}(\mathrm{mg})$ & $1 \cdot 73$ & 0.02 & 1.66 & 0.02 & $1 \cdot 79$ & 0.04 & 1.96 & 0.07 & $1 \cdot 89$ & $0 \cdot 11$ & 0.03 \\
\hline Vitamin $\mathrm{B}_{2}(\mathrm{mg})$ & $2 \cdot 22$ & 0.02 & $2 \cdot 13$ & 0.02 & $2 \cdot 32$ & 0.05 & $2 \cdot 55$ & 0.08 & $2 \cdot 48$ & $0 \cdot 14$ & $<0.001$ \\
\hline Vitamin $B_{6}(\mathrm{mg})$ & $1 \cdot 84$ & 0.03 & $1 \cdot 73$ & 0.04 & 1.92 & 0.04 & $2 \cdot 18$ & 0.09 & $2 \cdot 25$ & 0.14 & $<0.001$ \\
\hline Vitamin $B_{12}(\mu \mathrm{g})$ & $5 \cdot 19$ & 0.08 & 5.02 & 0.09 & $5 \cdot 46$ & $0 \cdot 17$ & $5 \cdot 66$ & 0.32 & 5.59 & 0.59 & 0.29 \\
\hline Niacin (mg) & $23 \cdot 19$ & 0.21 & $22 \cdot 61$ & 0.24 & $23 \cdot 87$ & 0.44 & $25 \cdot 18$ & 0.88 & $24 \cdot 53$ & $1 \cdot 45$ & $0 \cdot 18$ \\
\hline Folate $(\mu \mathrm{g})$ & $403 \cdot 46$ & $4 \cdot 55$ & $378 \cdot 20$ & $4 \cdot 12$ & $420 \cdot 21$ & $11 \cdot 32$ & $502 \cdot 16$ & $24 \cdot 16$ & 478.94 & $32 \cdot 28$ & $<0.001$ \\
\hline Calcium (mg) & 994.09 & $10 \cdot 61$ & 970.49 & $13 \cdot 35$ & $1038 \cdot 57$ & 26.09 & $1063 \cdot 64$ & $34 \cdot 19$ & $1009 \cdot 73$ & 61.94 & 0.34 \\
\hline Magnesium (mg) & $248 \cdot 46$ & $1 \cdot 61$ & $232 \cdot 43$ & $1 \cdot 74$ & $258 \cdot 45$ & 3.63 & $290 \cdot 81$ & $6 \cdot 23$ & $341 \cdot 88$ & $10 \cdot 54$ & $<0.001$ \\
\hline Potassium (mg) & $2446 \cdot 93$ & $17 \cdot 90$ & $2446 \cdot 36$ & $22 \cdot 38$ & $2443 \cdot 72$ & $42 \cdot 93$ & $2495 \cdot 54$ & $63 \cdot 40$ & $2362 \cdot 49$ & $105 \cdot 33$ & 0.33 \\
\hline Phosphorus (mg) & $1353 \cdot 88$ & $8 \cdot 34$ & $1320 \cdot 21$ & $10 \cdot 12$ & $1405 \cdot 52$ & $21 \cdot 70$ & $1443 \cdot 85$ & $27 \cdot 68$ & $1438 \cdot 30$ & 53.03 & 0.03 \\
\hline Iron (mg) & $16 \cdot 29$ & $0 \cdot 17$ & $15 \cdot 07$ & $0 \cdot 15$ & $16 \cdot 62$ & $0 \cdot 30$ & $20 \cdot 70$ & 0.81 & $22 \cdot 51$ & $1 \cdot 52$ & $<0.001$ \\
\hline Sodium (mg) & $3537 \cdot 86$ & $22 \cdot 91$ & 3555.03 & $30 \cdot 19$ & $3588 \cdot 63$ & $52 \cdot 34$ & 3372.54 & $61 \cdot 91$ & $3474 \cdot 01$ & $129 \cdot 50$ & 0.08 \\
\hline
\end{tabular}

WG, whole grains; NHANES, National Health and Nutrition Examination Survey; HEI, Healthy Eating Index; RAE, retinol activity equivalents; AT, $\alpha$-tocopherol. ${ }^{*}$ Estimates were adjusted for age, gender, ethnicity and total energy intake. Models for total energy did not include total energy intake.

$+W G$ was defined according to the new definition that excludes bran.

intake should be encouraged in children and adolescents to help them meet the fibre requirement.

All age groups showed a significant increase in magnesium with the consumption of WG. Magnesium is an essential cofactor for over 300 metabolic reactions. In adults, higher intake is associated with an inverse risk of type 2 diabetes ${ }^{(66)}$ and metabolic syndrome ${ }^{(67)}$. One of the few studies conducted in children suggested that magnesium deficiency is associated with insulin resistance in obese children ${ }^{(68)}$. Adolescents appear at greatest risk for low magnesium intake $^{(64)}$. In the present study, mean intake by adolescents failed to reach the DRI (separate data by gender not shown).

Vitamin E, calcium and potassium are the remaining shortfall nutrients in the diets of children. Vitamin $\mathrm{E}$ is found in WG; however, only $5 \%^{(69)}$ to $21 \%{ }^{(70)}$ of vitamin $\mathrm{E}$ is retained when wheat is processed. Adolescents were the only group that showed improved vitamin E intake with increased WG consumption and their mean intake did not approach the DRI of $15 \mathrm{mg} / \mathrm{d}$ for male and female adolescents aged $14-18$ years ${ }^{(35)}$. Increased consumption of WG by adults was associated with increased intake of vitamin $\mathrm{E}^{(71)}$; however, that study included dietary supplements in the nutrient analysis. Of the other shortfall nutrients, only potassium showed a marginal increase with increased consumption of WG in children aged 6-12 years. It may have been that WG consumption was not high enough to influence intake of vitamin $\mathrm{E}$, calcium and potassium, since WG are not good sources of these nutrients. Foods such as low-fat dairy, fruit, vegetables, nuts and oils should be encouraged along with WG in the diet to improve the intake of these micronutrients.

The present study had several limitations. NHANES collects cross-sectional data and, therefore, causal inferences cannot be drawn. Twenty-four-hour dietary recalls are subject to under- or over-reporting of energy and examiner effects ${ }^{(72)}$; single $24 \mathrm{~h}$ dietary recalls may not accurately reflect the usual dietary intake patterns of participants. Although $2 \mathrm{~d}$ recalls are now available from NHANES, only one day was used from the 2003-2004 data set; therefore, data from that reporting period would be comparable with data from the other survey years (1999-2002). Parents reported or assisted with the $24 \mathrm{~h}$ recalls of children aged 2-11 years; parents can often report accurately what children eat in the home ${ }^{(73)}$, but may not 
know what their children eat outside the home ${ }^{(74)}$, which could result in reporting errors ${ }^{(75)}$. With a large sample size, $24 \mathrm{~h}$ recalls produce reasonably accurate group estimates of nutrient intake ${ }^{(76)}$. The strengths of the study included a nationally representative sample with a large sample size and use of the new definition of WG.

Overall consumption of WG in children and adolescents was low; however, diet quality and nutrient intake were significantly improved with increasing consumption of WG. WG consumption for children, adolescents and their parents should be encouraged by health professionals, especially registered dietitians.

There is a paucity of published research looking at WG consumption in children. Recommendations for WG intake are based on findings in adults, which have been extrapolated to children and adolescents. Few studies have looked at ethnic and socio-economic influences on WG consumption in these age groups ${ }^{(35)}$. Barriers to WG intake in children and adolescents have not been widely studied ${ }^{(45)}$, and only recently have results from interventions to increase consumption of WG in children been published ${ }^{(55)}$. Nutrition education programmes that increase awareness, health benefits and consumption of WG should be made more widely available. Finally, it is important to quantify the effect that WG consumption has on health parameters, such as weight, in children.

\section{Acknowledgements}

This research was supported by funds from the Kellogg's Corporate Citizenship Fund. Partial support was received from the US Department of Agriculture (USDA) Hatch Projects 940-36-3104 Project no. 93673 and LAB 93676 no. 0199070 and a USDA/ARS (Agricultural Research Service)specific Cooperative Agreement no. 58-6250-6-003. This work is a publication of the USDA/ARS Children's Nutrition Research Center, Department of Pediatrics, Baylor College of Medicine in Houston, TX, USA, and was also funded in part with federal funds from the USDA/ARS under Cooperative Agreement no. 58-6250-6-003. The contents of this publication do not necessarily reflect the views or policies of the USDA, nor does the mention of trade names, commercial products, or organizations imply endorsement from the US government. The sponsors had no role in the design and conduct of the study; the collection, management, analysis and interpretation of the data; or the preparation and approval of the manuscript. The authors have no conflicts of interest to declare. C.E.O'N. directed implementation and was the principal author. T.A.N. conceptualized the study; helped with the editing and interpretation of the results. M.Z. and R.K. helped with the editing. S.S.C. conducted the statistical analyses. The authors thank Bee Wong for help with the literature and Pamelia Harris for formatting the manuscript.

\section{References}

1. Slavin J (2004) Whole grains and human health. Nutr Res Rev 17, 99-110

2. Slavin JL, Jacobs D, Marquart L et al. (2001) The role of whole grains in disease prevention. J Am Diet Assoc 101, 780-785.

3. American Association of Cereal Chemists (1999) International definition of whole grain. http://www.aaccnet.org/ definitions/wholegrain.asp (accessed October 2009).

4. US Food and Drug Administration (2006) Guidance for industry and FDA staff: whole grain label statements (draft guidance). http://www.fda.gov/Food/GuidanceCompliance RegulatoryInformation/GuidanceDocuments/FoodLabeling Nutrition/ucm059088.htm (accessed October 2009).

5. Franz M \& Sampson L (2006) Challenges in developing a whole grain database: definitions, methods, and quantification. J Food Compost Anal 19, S38-S44.

6. Jones JM, Reicks M, Adams J et al. (2002) The importance of promoting a whole grain foods message. J Am Coll Nutr 21, 293-297.

7. Jensen MK, Koh-Banerjee P, Hu FB et al. (2004) Intakes of whole grains, bran, and germ and the risk of coronary heart disease in men. Am J Clin Nutr 80, 1492-1499.

8. Steffen LM, Jacobs DR Jr, Stevens J et al. (2003) Associations of whole-grain, refined-grain, and fruit and vegetable consumption with risks of all-cause mortality and incident coronary artery disease and ischemic stroke: the Atherosclerosis Risk in Communities (ARIC) Study. Am J Clin Nutr 78, 383-390.

9. Wang L, Gaziano JM, Liu S et al. (2007) Whole- and refinedgrain intakes and the risk of hypertension in women. $\mathrm{Am} \mathrm{J}$ Clin Nutr 86, 472-479.

10. de Munter JS, Hu FB, Spiegelman D et al. (2007) Whole grain, bran, and germ intake and risk of type 2 diabetes: a prospective cohort study and systematic review. PLoS Med 4, e261.

11. Steffen LM, Jacobs DR Jr, Murtaugh MA et al. (2003) Whole grain intake is associated with lower body mass and greater insulin sensitivity among adolescents. Am J Epidemiol 158, 243-250.

12. Good CK, Holschuh N, Albertson AM et al. (2008) Whole grain consumption and body mass index in adult women: an analysis of NHANES 1999-2000 and the USDA pyramid servings database. J Am Coll Nutr 27, 80-87.

13. Larsson SC, Giovannucci E, Bergkvist L et al. (2005) Whole grain consumption and risk of colorectal cancer: a populationbased cohort of 60,000 women. Br J Cancer 92, 1803-1807.

14. Schatzkin A, Mouw T, Park Y et al. (2007) Dietary fiber and whole-grain consumption in relation to colorectal cancer in the NIH-AARP Diet and Health Study. Am J Clin Nutr 85, $1353-1360$.

15. Jacobs DR Jr \& Steffen LM (2003) Nutrients, foods, and dietary patterns as exposures in research: a framework for food synergy. Am J Clin Nutr 78, Suppl. 3, 508S-513S.

16. United States Department of Agriculture, Dietary Guidelines Advisory Committee, Agricultural Research Service (2005) Report of the Dietary Guidelines Advisory Committee on the Dietary Guidelines for Americans 2005: To the Secretary of Health and Human Services and the Secretary of Agriculture. http://www.health.gov/DietaryGuidelines/ dga2005/report/default.htm (accessed July 2008).

17. United States Department of Agriculture (modified 2010) MyPyramid Website. http://www.mypyramid.gov (accessed July 2008).

18. Gidding SS, Dennison BA, Birch LL et al. (2005) Dietary recommendations for children and adolescents: a guide for practitioners: consensus statement from the American Heart Association. Circulation 112, 2061-2075.

19. Kantor LS, Variyam JN, Allshouse JE et al. (2001) Choose a variety of grains daily, especially whole grains: a challenge for consumers. J Nutr 131, Suppl., S473-S486. 
20. Harnack L, Walters SA \& Jacobs DR Jr (2003) Dietary intake and food sources of whole grains among US children and adolescents: data from the 1994-1996 Continuing Survey of Food Intakes by Individuals. J Am Diet Assoc 103, 1015-1019.

21. Cook AJ \& Friday JE (2005) CNRG Table Set 3.O: Pyramid Servings Intakes in the United States 1999-2002, 1 Day. Beltsville, MD: US Department of Agriculture, Agricultural Research Service; available at http://www.ba.ars.usda.gov/ cnrg

22. De Moura FF, Lewis KD \& Falk MC (2009) Applying the FDA definition of whole grains to the evidence for cardiovascular disease health claims. J Nutr 139, Suppl., S2220-S2226.

23. Jacobs DR Jr, Meyer KA, Kushi LH et al. (1998) Whole-grain intake may reduce the risk of ischemic heart disease death in postmenopausal women: the Iowa Women's Health Study. Am J Clin Nutr 68, 248-257.

24. US Food and Drug Administration (1999) Health claim notification for whole grain foods. http://www.fda.gov/ ohrms/dockets/dailys/01/Sep01/091901/let0003.pdf (accessed July 2008).

25. Friday JE \& Bowman SA (2006) MyPyramid Equivalents Database for USDA Survey Food Codes, 1994-2002, version 1.0. Beltsville, MD: US Department of Agriculture, Agricultural Research Service; available at http://www.ars. usda.gov/ba/bhnrc/fsrg/

26. Bowman SA, Friday JE \& Mosfegh A (2008) MyPyramid Equivalents Database, 2.0 for USDA Survey Foods, 2003-2004. Beltsville, MD: US Department of Agriculture, Agricultural Research Service; available at http://www.ars. usda.gov/ba/bhnrc/fsrg/

27. Centers for Disease Control and Prevention (2008) National Health and Nutrition Examination Survey. http:// www.cdc.gov/nchs/nhanes.htm (accessed July 2009).

28. Centers for Disease Control and Prevention (2004) NHANES analytical guidelines. http://www.cdc.gov/nchs/ data/nhanes/nhanes_03_04/nhanes_analytic_guidelines_ dec_2005.pdf (accessed July 2008).

29. Jonnalagadda SS, Mitchell DC, Smiciklas-Wright $\mathrm{H}$ et al. (2000) Accuracy of energy intake data estimated by a multiple-pass, 24-hour dietary recall technique. J Am Diet Assoc 100, 303-308.

30. National Center for Health Statistics (2004) NHANES MEC In-Person Dietary Interviewers Procedures Manual. http:// www.cdc.gov/nchs/data/nhanes/nhanes_03_04/DIETARY_ MEC.pdf (accessed July 2008).

31. Cook A, Friday JE \& Subar AF (2004) Dietary Source Nutrient Database for USDA Food Codes. Beltsville, MD: US Department of Agriculture, Agricultural Research Service; available at http://www.ars.usda.gov/ba/bhnrc/fsrg/

32. US Department of Agriculture (2004) USDA Food and Nutrient Database for Dietary Studies, 1.O. Beltsville, MD: Agricultural Research Service, Food Surveys Research Group.

33. US Department of Agriculture (2006) USDA Food and Nutrient Database for Dietary Studies, 2.O. Beltsville, MD: Agricultural Research Service, Food Surveys Research Group.

34. Institute of Medicine (2001) Dietary Reference Intakes for Vitamin A, Vitamin K, Arsenic, Boron, Chromium, Copper, Iodine, Iron, Manganese, Molybdenum, Nickel, Silicon, Vanadium, and Zinc. Washington, DC: National Academy Press.

35. Institute of Medicine (2000) Dietary Reference Intakes for Vitamin C, Vitamin E, Selenium, and Carotenoids. Washington, DC: National Academy Press.

36. US Department of Agriculture (2006) USDA Database of Vitamin A ( $m c g$ RAE) and Vitamin E ( $m g$ AT) for National Health and Nutrition Examination Survey 1999-2000.
Beltsville, MD: Agricultural Research Service, Food Surveys Research Group.

37. Guenther PM, Reedy J, Krebs-Smith SM et al. (2007) Development and evaluation of the Healthy Eating Index2005: technical report. http://www.cnpp.usda.gov/Healthy EatingIndex.htm (accessed July 2008).

38. US Department of Agriculture, Center for Nutrition Policy and Promotion (2009) Healthy Eating Index-2005. Development and evaluation: technical report support files. http://www.cnpp.usda.gov/HealthyEatingIndex-2005report. htm (accessed July 2009).

39. Cleveland LE, Moshfegh AJ, Albertson AM et al. (2000) Dietary intake of whole grains. J Am Coll Nutr 19, Suppl. 3, S331-S338.

40. Lin BH \& Yen ST (2007) The US Grain Consumption Landscape: Who Eats Grain, in What Form, Where, and How Much? Economic Research Report no. ERR-50. Washington, DC: USDA Economic Research Service; available at http://www.ers.usda.gov/Publications/ERR50/

41. Newby PK, Maras J, Bakun P et al. (2007) Intake of whole grains, refined grains, and cereal fiber measured with 7-d diet records and associations with risk factors for chronic disease. Am J Clin Nutr 86, 1745-1753.

42. Baranowski T, Watson K, Missaghian M et al. (2008) Social support is a primary influence on home fruit, $100 \%$ juice, and vegetable availability. J Am Diet Assoc 108, 1231-1235.

43. Haerens L, Craeynest M, Deforche B et al. (2008) The contribution of psychosocial and home environmental factors in explaining eating behaviours in adolescents. Eur J Clin Nutr 62, 51-59.

44. Hanson NI, Neumark-Sztainer D, Eisenberg ME et al. (2005) Associations between parental report of the home food environment and adolescent intakes of fruits, vegetables and dairy foods. Public Health Nutr 8, 77-85.

45. Faith MS, Scanlon KS, Birch LL et al. (2004) Parent-child feeding strategies and their relationships to child eating and weight status. Obes Res 12, 1711-1722.

46. Patrick H, Nicklas TA, Hughes SO et al. (2005) The benefits of authoritative feeding style: caregiver feeding styles and children's food consumption patterns. Appetite $\mathbf{4 4}$, 243-249.

47. Burgess-Champoux TL, Rosen R, Marquart L et al. (2008) The development of psychosocial measures for wholegrain intake among children and their parents. J Am Diet Assoc 108, 714-717.

48. Patrick H \& Nicklas TA (2005) A review of family and social determinants of children's eating patterns and diet quality. J Am Coll Nutr 24, 83-92.

49. Burgess-Champoux T, Marquart L, Vickers Z et al. (2006) Perceptions of children, parents, and teachers regarding whole-grain foods, and implications for a school-based intervention. J Nutr Educ Behav 38, 230-237.

50. Befort C, Kaur H, Nollen N et al. (2006) Fruit, vegetable, and fat intake among non-Hispanic black and nonHispanic white adolescents: associations with home availability and food consumption settings. J Am Diet Assoc 106, 367-373.

51. Larson NI, Story M, Wall M et al. (2006) Calcium and dairy intakes of adolescents are associated with their home environment, taste preferences, personal health beliefs, and meal patterns. J Am Diet Assoc 106, 1816-1824.

52. Li J \& Wang Y (2008) Tracking of dietary intake patterns is associated with baseline characteristics of urban low-income African-American adolescents. J Nutr 138, 94-100.

53. Nicklas TA (1995) Dietary studies of children: the Bogalusa Heart Study experience. J Am Diet Assoc 95, 1127-1133.

54. Singer MR, Moore LL, Garrahie EJ et al. (1995) The tracking of nutrient intake in young children: the Framingham Children's Study. Am J Public Health 85, 1673-1677. 
55. Burgess-Champoux TL, Chan HW, Rosen R et al. (2008) Healthy whole-grain choices for children and parents: a multi-component school-based pilot intervention. Public Health Nutr 11, 849-859.

56. US Department of Agriculture, Food and Nutrition Service (2007) Incorporating the 2005 Dietary Guidelines for Americans into school meals. http://www.fns.usda.gov/ cnd/Governance/Policy-Memos/2008/SP_04-2008-OS.pdf (accessed July 2008).

57. US Department of Agriculture, Food and Nutrition Service (2000) Menu Planning for the National School Lunch Program. http://www.fns.usda.gov/cnd/menu/menu_ planning.doc (accessed July 2008).

58. Institute of Medicine (2007) Nutrition standards for foods in schools: leading the way toward healthier youth. http:// www.iom.edu/Object.File/Master/42/628/fact\%20sheet.pdf (accessed July 2008).

59. Kennedy E (2008) Putting the pyramid into action: the Healthy Eating Index and Food Quality Score. Asia Pac J Clin Nutr 17, Suppl. 1, 70-74.

60. Feskanich D, Rockett HR \& Colditz GA (2004) Modifying the Healthy Eating Index to assess diet quality in children and adolescents. J Am Diet Assoc 104, 1375-1383.

61. US Department of Agriculture, Agricultural Research Service (2004) Nutrient intakes: mean amounts consumed per individual, one day, 2003-2004. http://www.ars.usda. gov/SP2UserFiles/Place/12355000/pdf/0304/Table_1_NIF. pdf (accessed July 2008).

62. Institute of Medicine (2005) Dietary Reference Intakes for Energy, Carbohydrate, Fiber, Fat, Fatty Acids, Cholesterol, Protein, and Amino Acids (Macronutrients). Washington, DC: National Academy Press; available at http://www.nap. edu/books/0309085373/html

63. Williams CL \& Strobino BA (2008) Childhood diet, overweight, and CVD risk factors: the Healthy Start project. Prev Cardiol 11, 11-20.

64. Lee WT, Ip KS, Chan JS et al. (2008) Increased prevalence of constipation in pre-school children is attributable to under-consumption of plant foods: a community-based study. J Paediatr Child Health 44, 170-175.

65. Loening-Baucke V (1995) Functional constipation. Semin Pediatr Surg 4, 26-34.

66. Song Y, Manson JE, Buring JE et al. (2004) Dietary magnesium intake in relation to plasma insulin levels and risk of type 2 diabetes in women. Diabetes Care 27, 59-65.

67. McKeown NM, Jacques PF, Zhang XL et al. (2008) Dietary magnesium intake is related to metabolic syndrome in older Americans. Eur J Nutr 47, 210-216.

68. Huerta MG, Roemmich JN, Kington ML et al. (2005) Magnesium deficiency is associated with insulin resistance in obese children. Diabetes Care 28, 1175-1181.

69. Liu S (2002) Intake of refined carbohydrates and whole grain foods in relation to risk of type 2 diabetes mellitus and coronary heart disease. J Am Coll Nutr 21, 298-306.

70. Truswell AS (2002) Cereal grains and coronary heart disease. Eur J Clin Nutr 56, 1-14.

71. Koh-Banerjee P, Franz M, Sampson L et al. (2004) Changes in whole-grain, bran, and cereal fiber consumption in relation to 8-y weight gain among men. Am J Clin Nutr 80, $1237-1245$.

72. Johnson RK, Driscoll P \& Goran MI (1996) Comparison of multiple-pass 24-hour recall estimates of energy intake with total energy expenditure determined by the doubly labeled water method in young children. J Am Diet Assoc 96, 1140-1144.

73. Basch CE, Shea S, Arliss R et al. (1990) Validation of mothers' reports of dietary intake by four to seven year-old children. Am J Public Health 80, 1314-1317.

74. Baranowski T, Sprague D, Baranowski JH et al. (1991) Accuracy of maternal dietary recall for preschool children. J Am Diet Assoc 91, 669-674.

75. Schoeller DA (1990) How accurate is self-reported dietary energy intake? Nutr Rev 48, 373-379.

76. Hennekens CH \& Buring J (1987) Epidemiology in Medicine. Baltimore, MD: Lippincott, Williams \& Wilkins. 